\begin{tabular}{|c|l|}
\hline Title & A bsence of the hump in the density of states of percolating clusters \\
\hline Author(s) & Y akubo K.; Nakayama, T. \\
\hline Citation & $\begin{array}{l}\text { PHY SICAL REVIEW B, 36(16), 8933-8936 } \\
\text { https://doi.org/L0.1103/PhysRevB.36.8933 }\end{array}$ \\
\hline Issue Date & 1987-12-01 \\
\hline Doc URL & http://hdl.handle.net/2115/5657 \\
\hline Rights & Copyright $\odot$ 1987 A merican Institute of Physics \\
\hline Type & article \\
\hline File Information & PRB36-16.pdf \\
\hline
\end{tabular}

Instructions for use 


\title{
Absence of the hump in the density of states of percolating clusters
}

\author{
K. Yakubo and T. Nakayama \\ Department of Applied Physics, Hokkaido University, Sapporo 060, Japan
}

(Received 10 August 1987)

\begin{abstract}
The vibrational densities of states of two-dimensional percolating clusters with very large cluster size are investigated using a novel numerical method. It is confirmed that the fracton density of states is proportional to $\omega^{1 / 3}$ in accord with the conjecture of Alexander and Orbach. We find that there is no notable steepness or hump in the phonon-fracton crossover region, in conflict with the prediction of the effective-medium theory. It is shown that the fracton modes are localized in "peninsulas" in percolating clusters.
\end{abstract}

Over the past few years the nature of vibrational modes in percolating clusters has received a great deal of attention. ${ }^{1-6}$ In particular, the scaling argument for the density of states ${ }^{1}$ (DOS) has made much progress in understanding this problem. Alexander and Orbach ${ }^{1}$ have suggested that the DOS's of percolating clusters in all Euclidean dimensions $d(d \geq 2)$ obey the universal law $g(\omega) \propto \omega^{1 / 3}$ in the regime above the characteristic frequency $\omega_{c}$. Vibrational modes in this regime are called "fractons." The phonon regime also exists in which the DOS follows the conventional Debye law $g(\omega) \propto \omega^{d-1}$. Experimental results ${ }^{7-11}$ indicate that no discontinuity or singularity exists in the phonon-fracton crossover region of the DOS. The effective-medium theory ${ }^{2,3}$ predicts that the DOS increases more "steeply" in the crossover region than that of the Debye law. Though the effective-medium theory presents the correct physical results for lowfrequency properties of the system, the theory often leads to incorrect results for the higher-frequency properties. For instance, this theory shows that the DOS in the fracton regime becomes constant for $2 \geq d \geq 4$, contrary to the Alexander-Orbach conjecture. It has been suggested, ${ }^{2}$ from degrees-of-freedom arguments, that there should be a "hump" in the crossover region. Some experimental results $^{7-10}$ on amorphous solids show the existence of "steepness" or "hump" in the crossover region of the DOS. A recent experimental study 11 of the low-frequency inelastic light scattering in superionic borate glass with the fractal structure shows no steepness, or does it show the hump in the crossover region.

The numerical study on the density of states of threedimensional percolating clusters has been performed by Grest and Webman. ${ }^{12}$ Although their results seem to support the existence of the steepness in this region, their percolating clusters consist of relatively small site numbers $(N \lesssim 2200)$. As a result, finite-size effects influence the result in the low-frequency regime. Thus the numerical study of the density of states for very large clusters is necessary in order to obtain correct insight into the vibrational spectra of percolating clusters. ${ }^{2}$

In this Rapid Communication, we present a numerical study of the frequency spectra and mode patterns of vibrations in two-dimensional percolating clusters with large site numbers using a novel numerical method. ${ }^{13}$ It is confirmed that there are two distinct frequency regimes in the DOS characterized by $\omega_{c}$. In addition, it is found that no notable steepness or hump exists in the phonon-fracton crossover region. We also present the mode patterns of fractons, which show the patterns peculiar to percolating clusters.

Consider a site-percolating cluster consisting of $N$ particles with unit mass and linear springs connecting two nearest-neighbor atoms. The equations of motion of the atoms are

$$
\ddot{u}_{n}(t)+\sum_{m} K_{n m} u_{m}(t)=0
$$

where $u_{n}$ is the displacement of the atom on the $n$th site. The force constant is taken to be $K_{n m}=0$ if either sites $n$ or $m$ are unoccupied, and $K_{n m}=1$ otherwise. The displacement $u_{n}$ has only one component. Such a simplification does not affect the understanding of the intrinsic nature of the dynamics of the present system. In an ordinary numerical method one must diagonalize the dynamical matrix $\left[K_{n m}\right]$ in order to calculate the eigenvalue $\omega_{\lambda}$ and its eigenvector. This conventional method requires a large amount of computer time as the cluster size $N$ becomes large. In addition, the standard numerical routine for the diagonalization yields poor accuracy for the very-low-frequency eigenvalues. This is unsuitable for our problem because the cluster size $N$ must be taken very large to avoid the ambiguity in the low-frequency region.

The numerical technique employed here is based on the idea that the eigenfrequency of the system satisfies the resonance condition when applying the periodic external force with frequency $\omega$ to the system. This technique has been developed by Williams and Maris ${ }^{13}$ and applied to the problem of a harmonic system with a mass disorder. The method has the following advantages: (i) One can apply this algorithm to a very large system $\left(N>10^{5}\right)$; (ii) it is possible to calculate quite accurately the DOS in the low-frequency regime; (iii) the magnitude of the DOS at any frequency can be obtained by sweeping the resonant frequency.

A random force given by $F_{0} \cos \left(\phi_{n}\right) \cos (\omega t)$ is applied to each atom at rest and with zero displacement at $t=0$. Here $F_{0}$ is constant and $\phi_{n}$ is a random quantity. After the time interval $t$, the system is given the finite energy $E(\omega, t)$ by the forced vibration. If we choose $t$ appropriately, the density of states of the percolating-cluster sys- 
tem can be related to the total energy as given by ${ }^{13}$

$$
g(\omega)=\frac{8\langle E(\omega, t)\rangle}{\pi t F_{0}^{2} N},
$$

where $\langle E(\omega, t)\rangle$ is the averaged total energy of the system. The accuracy of the density of states obtained in this way is independent of the frequency $\omega$, but depends on the number of vibrational modes belonging to the frequencies in the resonant width $\Delta \omega$. Mode patterns can also be obtained by applying iteratively the external periodic force proportional to the displacements of atoms at each step.

We can calculate numerically the averaged energy $\langle E(\omega, t)\rangle$ or displacements $u_{n}$ from the difference equation corresponding to the equation of motion for the forced vibration. The array-processing supercomputer has been utilized on actual calculations. The algorithm is exceedingly suitable to vectorize the computer program. Indeed, we have achieved the ratio of vectorization more than $99 \%$.

The accuracy of these calculations is checked by comparing the density of states obtained for the regular twodimensional square lattice $(100 \times 100)$ with the corresponding exact solution. ${ }^{14}$ The numerical results agree well with the exact solution; especially the agreement in the very-low-frequency regime, which is excellent. The results for the DOS of percolating clusters are shown in Fig. 1. Figure 1(a) shows the DOS of two-dimensional site-percolating clusters for the percolation threshold $p_{c}(=0.593)$. The result is averaged over seven clusters of size $N=3714$ to 4854 formed on $100 \times 100$ square lattices. The large cluster size makes it possible to clarify the



FIG. 1. The DOS's of two-dimensional site percolating clusters. (a) The DOS is averaged over seven clusters (size $N=3714$ to 4854$)$ with $p_{c}(=0.593)$ formed on $100 \times 100$ square lattices. (b) The concentration $p$ is taken $p=0.67$. The cluster is formed on a $700 \times 700$ square lattice. The cluster size is $N=317672$. very-low-frequency vibrational behavior. The DOS is closely proportional to $\omega^{1 / 3}$ for $\omega \ll 1$ as predicted by Alexander and Orbach. ${ }^{1}$ This implies the fracton dimensionality $\overline{\bar{d}}$ to be very close to $\frac{4}{3}$ for the percolating cluster. Since the system is not fractal on a length scale shorter than the wavelength corresponding to $\omega=1$, the DOS does not exhibit the $\omega^{1 / 3}$ dependence above $\omega=1$.

The density of states of a two-dimensional sitepercolating cluster with $p=0.67$ is shown in Fig. 1(b). This percolating cluster is formed on a $700 \times 700$ square lattice with the cluster size $N=317672$. In the frequency region $\omega_{c} \ll \omega \ll 1$, the DOS is clearly proportional to $\omega^{1 / 3}$. The characteristic frequency $\omega_{c}$ corresponds to the wavelength equal to the percolation correlation length $\xi_{p}$. Percolating clusters can be regarded as a uniform system on a longer length scale than $\xi_{p} .{ }^{15}$ The correlation length $\xi_{p}$ depends on the concentration $p$ as $\left|p-p_{c}\right|^{-v}$, where the critical exponent $v$ is a positive constant. ${ }^{15}$ Therefore the DOS in the lower-frequency regime rather than $\omega_{c}$ should be given by the conventional Debye law $g(\omega) \propto \omega^{d-1}$. Our result is consistent with this argument because the frequency dependence of the DOS for lower frequencies $\left(\omega \ll \omega_{c}\right)$ clearly obeys the law $g(\omega) \propto \omega$. Vibrational excitations in this frequency regime behave as phonons.

The region in the vicinity of $\omega_{c}$ is the crossover region between phonons and fractons [Fig. 1(b)]. We see that the density of states is smoothly connected in this region. In contrast with the prediction of the effective-medium theory ${ }^{3}$ or the numerical work by Grest and Webman, ${ }^{12}$ our results do not exhibit a notable steepness or the presence of a hump in the vicinity of $\omega_{c}$. We have checked this point for three-dimensional percolating clusters with $p>p_{c}$ and found that no steepness or hump exists in the crossover region. Therefore our results indicate the absence of steepness or a hump in the phonon-fracton crossover region of the DOS and are in accord with the selfconsistent theory of Loring and Mukamel. ${ }^{16}$

We should mention the experimental results ${ }^{7-10}$ that do exhibit steepness or a hump in the density of states. These results are on amorphous solids composed of covalent bonding, where both the bond-bending and bondstretching force constants are important for determining the DOS. There is a theoretical suggestion, ${ }^{5}$ for a system with bond-bending as well as bond-stretching force constants, that the hump appears in the DOS under certain conditions. It seems, as discussed by Buchenau et al., ${ }^{9}$ that the observed hump can be attributed to some intrinsic modes peculiar to amorphous solids and is not related to the hump predicted by the effective-medium theory. ${ }^{3}$ In the case of superionic borate glass, ${ }^{11}$ the Coulomb central force is important in the dynamical property. Our numerical results for the model with one stretching force constant are consistent with the experimental results by Fontana, Rocca, and Fontana. ${ }^{11}$

The snapshots of vibrational mode patterns of twodimensional percolating clusters are shown in Figs. 2(a) and 2(b). Figure 2(a) shows the mode pattern of a percolating cluster with $p=0.70$ on a $100 \times 100$ square lattice with a free boundary condition. The cluster has the size $N=6815$. The displacements of atoms are expressed by 


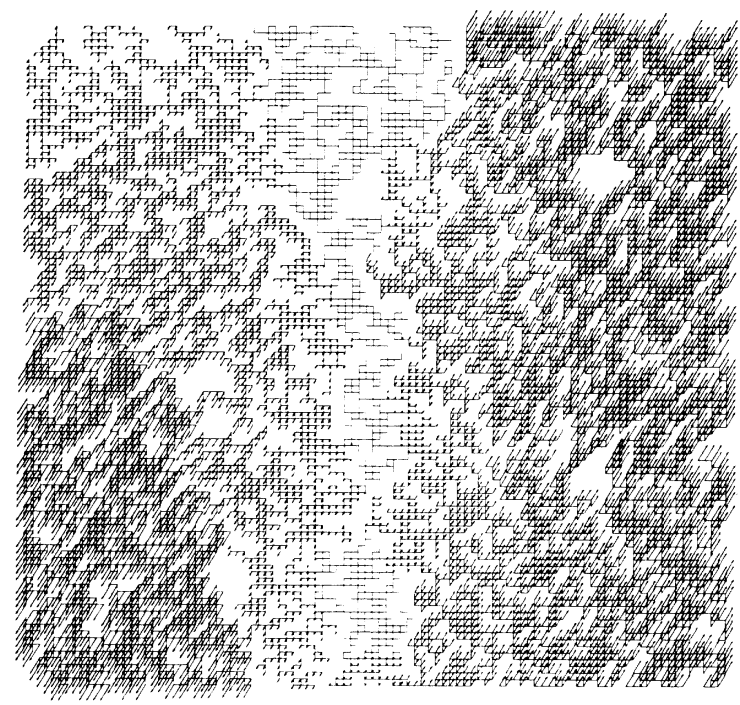

(a)

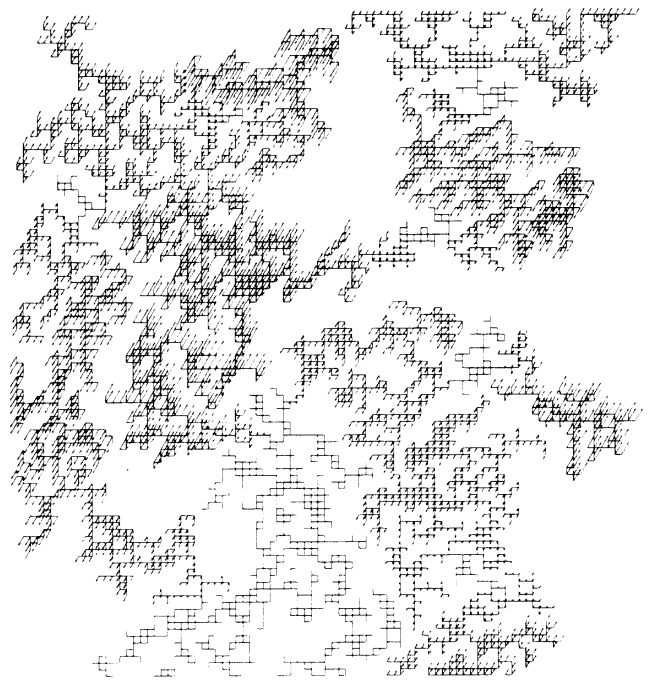

(b)

FIG. 2. The vibrational mode patterns of two-dimensional site percolating clusters (a) with the concentration $p=0.70$ (cluster size $N=6815$ ), and (b) with the percolation threshold $p_{c}$ (cluster size $N=3714$ ). These clusters are formed on a $100 \times 100$ square lattice. Both modes belong to the eigenfrequency $\omega=0.02$. The displacements of atoms are shown by oblique arrows. We see from (b) that the fracton mode is localized in "peninsulas."

oblique arrows. We see from Fig. 2(a) that the phonon mode $(\omega=0.02)$ is extended over the whole system. The mode pattern of a percolating cluster with $p=0.593\left(\omega_{c}\right.$ $=0)$ on a $100 \times 100$ square lattice is shown in Fig. 2(b). The cluster used here has the size $N=3714$. This mode belongs to the eigenfrequency $\omega=0.02$ and behaves as a fracton. One sees that fractons are localized in "peninsulas" in the percolating cluster. It has been suggested by the scaling argument ${ }^{17}$ that the fracton excitations are localized modes. This localization nature of fractons is numerically shown in the present study for the first time. There have been a number of reports ${ }^{18-20}$ about the plateau of thermal conductivities of topologically disordered systems such as amorphous solids. Our results on mode patterns will be useful in understanding the origin of the plateau in thermal conductivities. ${ }^{6}$

In conclusion, we have performed computer experiments of two-dimensional percolating clusters with a large particle size $\left(N=3 \times 10^{5}\right)$, in which the AlexanderOrbach conjecture has been confirmed numerically. It has become clear that two distinct frequency regions (phonon and fracton regimes) exist in the density of states of the percolating clusters with $p>p_{c}$. We have presented numerical evidence for the absence of notable steepness or a hump in the phonon-fracton crossover region of the DOS. We have suggested that the steepness or hump observed by experiments ${ }^{7-10}$ has its origin in the vibrational modes attributing to the bond-bending force ${ }^{5}$ and has nothing to do with the steepness predicted by the effective-medium theory.

We wish to thank K. Takasugi and S. Tanaka for their help in the computer programming. The Hokkaido University Computing Center is also acknowledged for the use of the supercomputer facilities.
${ }^{1}$ S. Alexander and R. Orbach, J. Phys. (Paris) Lett. 43, L625 (1982).

${ }^{2}$ R. Orbach, J. Stat. Phys. 36, 735 (1984); A. Aharony, S. Alexander, O. E. Wohlman, and R. Orbach, Phys. Rev. B 31, 2565 (1985); R. Orbach, Science, 231, 814 (1986).

${ }^{3}$ O. E. Wohlman, S. Alexander, R. Orbach, and K. W. Yu, Phys. Rev. B 29, 4588 (1984); B. Derrida, R. Orbach, and K. W. Yu, ibid. 29, 6645 (1984).

${ }^{4}$ A. Aharony, S. Alexander, O. E. Wohlman, and R. Orbach, Phys. Rev. Lett. 58, 132 (1987).

${ }^{5}$ S. Feng, Phys. Rev. B 32, 5793 (1985).

${ }^{6}$ S. Alexander, O. E. Wohlman, and R. Orbach, Phys. Rev. B
34, 2726 (1986).

${ }^{7}$ U. Buchenau, N. Nuecker, and A. J. Dianoux, Phys. Rev. Lett. 53, 2316 (1984).

${ }^{8}$ H. M. Rosenberg, Phys. Rev. Lett. 54, 704 (1985).

${ }^{9}$ U. Buchenau, M. Prager, N. Nuecker, A. J. Dianoux, N. Ahmad, and W. A. Phillips, Phys. Rev. B 34, 5665 (1986).

${ }^{10}$ A. J. Dianoux, J. N. Page, and H. M. Rosenberg, Phys. Rev. Lett. 58, 886 (1987).

${ }^{11}$ A. Fontana, F. Rocca, and M. P. Fontana, Phys. Rev. Lett. 58, 503 (1987).

${ }^{12}$ G. S. Grest and I. Webman, J. Phys. (Paris) Lett. 45, L1155 (1984). 
${ }^{13}$ M. L. Williams and H. J. Maris, Phys. Rev. B 31, 4508 (1985).

${ }^{14}$ W. A. Bowers and H. B. Rosenstock, J. Chem. Phys. 18, 1056 (1950).

${ }^{15}$ A. Aharony, J. Stat. Phys. 34, 931 (1984).

${ }^{16}$ R. F. Loring and S. Mukamel, Phys. Rev. B 34, 6582 (1986).

${ }^{17}$ R. Rammal and G. Toulouse, J. Phys. (Paris) Lett. 44, L13 (1983).
${ }^{18}$ A. C. Anderson, in Amorphous Solids Low-Temperature Properties, edited by W. A. Phillips (Springer-Verlag, Berlin, $1981)$, p. 65.

${ }^{19}$ J. E. Graebner, B. Golding, and L. C. Allen, Phys. Rev. B 34, 5696 (1986)

${ }^{20}$ J. E. Graebner and B. Golding, Phys. Rev. B 34, 5788 (1986). 